\title{
DESENVOLVIMENTO E AVALIAÇÃO DE UM SOFTWARE EDUCACIONAL PARA O ENSINO DE ENFERMAGEM PEDIÁTRICA
}

Zem-Mascarenhas SH, Cassiani SHB. Desenvolvimento e avaliação de um software educacional para o ensino de enfermagem pediátrica. Rev Latino-am Enfermagem 2001 novembro-dezembro; 9(6):13-8.

A informática vem sendo introduzida na educação em ritmo acelerado, obrigando educadores e educandos a familiarizarem-se com essa tecnologia. Este estudo teve como objetivo o desenvolvimento e avaliação do software educacional "A criança e o medicamento". 0 programa utiliza recursos multimídia sendo composto por quatro tópicos: a criança, o medicamento, quem administra e administração do medicamento. A avaliação mostrou grande aceitabilidade do programa, recebendo conceito excelente para a maioria dos itens avaliados. Ressaltamos a importância do desenvolvimento de estratégias de ensino utilizando recursos computacionais para o ensino de enfermagem, capacitando os enfermeiros do próximo milênio.

DESCRITORES: software, enfermagem pediátrica, enfermagem-estudo e ensino, medicamentos

\section{DEVELOPMENT AND EVALUATION OF AN EDUCATIONAL SOFTWARE FOR PEDIATRIC NURSING EDUCATION}

Computer Science is being introduced in education at an accelerated pace, thus forcing educators and students to become familiarized with such technology. This study aimed at the development and evaluation of the educational software "Children and medication". The program utilizes multimedia resources in four topics: the child, medication, the person who administers and administration of medication. The evaluation showed a large acceptance of the program, with most of the items being rated as excellent. We emphasize the importance of the development of teaching strategies utilizing computer resources in nursing education and in the development of nurses for the new millenium.

KEY WORDS: software, pediatric nursing, nursing studying and teaching, medication

\section{DESARROLLO Y EVALUACIÓN DE UN SOFTWARE EDUCATIVO PARA LA ENSEÑANZA DE LA ENFERMERÍA PEDIÁTRICA}

La informática viene siendo introducida en la educación a ritmo acelerado, obligando a educadores y educandos a familiarizarse con esa tecnología. Esta investigación tuvo por objetivo el desarrollo y la evaluación del software educativo "El niño y el medicamento". El programa utiliza recursos multimedia y está compuesto por cuatro tópicos: el niño, el medicamento, quien lo administra y la administración del medicamento. La evaluación mostró aceptación del programa, y recibió concepto excelente en la mayor parte de los ítems evaluados. Resaltamos la importancia del desarrollo de estrategias de enseñanza utilizando recursos computacionales para la enseñanza de enfermería, capacitando los enfermeros del próximo milenio.

DESCRIPTORES: software, enfermería pediátrica, enfermería estudio y enseñanza, medicamentos

\footnotetext{
${ }^{1}$ Enfermeiro, Doutor em Enfermagem, endereço: Rua: Dr. Serafim Vieira de Almeida, 203 - Jardim Paraíso - CEP 13561-130 - São Carlos - São Paulo - Brasil, e-mail: silviazen@mzo.com.br; ${ }^{2}$ Enfermeiro, Professor Associado da Escola de Enfermagem de Ribeirão Preto da Universidade de São Paulo, Centro Colaborador da OMS para o desenvolvimento da pesquisa em enfermagem
} 


\section{INTRODUÇÃO}

As mudanças e evoluções tecnológicas não são mais consideradas em anos e sim em meses. Tecnologias apresentadas como novíssimas em termos de pesquisa de ponta tornam-se ultrapassadas em poucos meses. A cada minuto surge um novo hardware, um novo software, uma nova linguagem de programação, novos conceitos na área de informática, ou seja, as transformações são rápidas e constantes.

No âmbito escolar, o computador teve sua inserção na década de 50, com Skinner propondo uma máquina para ensinar, usando o conceito de instrução programada. Para tanto dividia-se 0 material instrucional em pequenos segmentos logicamente encadeados e denominados módulos, apresentados de forma impressa $^{(1)}$.

Os sistemas computacionais utilizados para o ensino são conhecidos como CAl (Computer Assisted Instruction) ou instrução assistida/auxiliada por computador. Esses sistemas têm como principal objetivo a transmissão de informações sobre um determinado assunto. A instrução assistida por computador pode ajudar o usuário a aumentar suas habilidades de trocar informações com 0 computador, de modo apropriado para a resolução de problemas e preparar os estudantes para seu futuro papel numa sociedade tecnológica.

Um programa Cai sobre medicamentos foi desenvolvido para 0 ensino da administração dos mesmos. Este programa foi concebido para ser utilizado como recurso auxiliar do ensino em programas de educação continuada, e programas formais de preparação de auxiliares, técnicos de enfermagem e enfermeiros. A autora destaca que uma das vantagens desse tipo de instrução é de permitir ao aluno programar seus momentos de estudo, aprendendo, assim, a aprender ${ }^{(2)}$.

Como o estudo acima citado, outras experiências estão acontecendo nas universidades brasileiras, o que reforça nossa crença de que a informática no ensino de enfermagem, como em outras áreas, é um caminho sem volta e que estamos apenas começando a desvendá-lo.

Dessa forma, espera-se que com o desenvolvimento de novos programas instrucionais, educadores e educandos tendam a utilizá-los cada vez mais, usufruindo das vantagens que eles podem oferecer ao ensino de enfermagem.

A informática pode ser utilizada pelos docentes e profissionais de enfermagem de várias maneiras como na construção de bases de dados informatizados, desde material bibliográfico, até aquelas aplicadas em diferentes atividades de enfermagem.

A experiência de construção de bases de dados informatizada é relatada por outros autores ${ }^{(3-4)}$, segundo as quais, a criação de uma base de dados particular e com conteúdo relacionado a uma área específica, apresenta certas vantagens em relação às bases de uso geral, sendo a principal delas o fato do autor conhecer sua estrutura e conteúdo.

Assim, a realização de estudos aprofundando questões relativas à aplicabilidade da informática na enfermagem, poderá subsidiar as escolas ao adotarem esta tecnologia em suas atividades didáticas. Buscando integrar a informática ao ensino de enfermagem e colaborar com o desenvolvimento de novas estratégias para a melhoria do processo ensino-aprendizagem, utilizando os recursos que essa tecnologia oferece, planejamos este estudo.

Por ser a enfermagem pediátrica nossa área de atuação, optamos por desenvolver um software educacional sobre administração de medicamentos porque, entre as técnicas pediátricas, esta é a de competência exclusiva da equipe de enfermagem, embora outros profissionais também a executem. Além disso, essa técnica é considerada uma das mais importantes, uma vez que as finalidades da hospitalização da criança, normalmente estão voltadas à terapêutica medicamentosa, principalmente com relação aos medicamentos injetáveis, visto que os demais podem ser administrados em casa pela mãe, pai ou responsável.

Outro aspecto que também deve ser considerado é o da formação de profissionais. Nossa experiência como docentes da área, mostrou-nos que os alunos sentem muita insegurança em relação à administração de medicamentos em pediatria, especialmente os injetáveis. 0 exposto, aliado à escassez de material didático sobre 0 tema, vem reforçar o motivo que nos levou a optar pelo desenvolvimento do software educacional "A criança e o medicamento".

\section{OBJETIVO}

Este estudo foi planejado tendo por objetivo avaliar a execução e a adequação de um software educacional, utilizando recursos de hipermídia, sobre a administração de medicamentos em pediatria.

\section{MÉTODOS}

Referencial pedagógico empregado

A concepção de aprendizagem aqui utilizada foi baseada nos estudos de Robert M. Gagné, que proporcionam diretrizes para as áreas de desenho instrucional e de eventos instrucionais. Os processos de aprendizagem podem estar sujeitos a influências do 
ambiente do aprendiz, portanto, determinados fatos podem ser produzidos de tal forma que afetem a motivação do estudante, sua atenção ou qualquer outro processo componente do ato total da aprendizagem ${ }^{(5)}$.

As fases de aprendizagem - fase de motivação, fase de apreensão, fase de aquisição, fase de retenção, fase de rememoração, fase de generalização, fase de desempenho, fase de feedback $^{(5)}$ - permearam todas as etapas de planejamento, desenvolvimento e avaliação do software educacional aqui descritas. A apresentação do conteúdo instrucional foi elaborada visando a utilização de uma série de recursos hipermídia para afetar os processos de aprendizagem, e as interações computador-usuário foram desenhadas com o objetivo de promover a aprendizagem.

Desenvolvimento do software educacional

Construímos o software utilizando o Modelo de três estágios para o desenvolvimento de Programas $C A \mathrm{I}^{(1)}$. Esse modelo é baseado nas diretrizes apresentadas por Gagné, e os três estágios são: planejamento inicial, planejamento e desenvolvimento do conteúdo instrucional, e avaliação e revisão.

Considerando o tema abordado no software de importância não só para estudantes ou profissionais de enfermagem, como também para pais e professores que atuam no ensino a crianças, procuramos desenvolver o conteúdo de forma clara e simples, sendo dividido em quatro tópicos: a criança, o medicamento, quem administra e a administração do medicamento. Para o desenvolvimento deste programa foi utilizado o software de autoria de programas multimídia Macromedia Director ${ }^{\circledR} 7$, que proporciona um método rápido para o desenvolvimento de infra-estrutura interativa através do uso de botões de controle.

Avaliação e revisão do software educacional

A avaliação de um software educacional é uma etapa de fundamental importância para que sejam assegurados que os objetivos e metas propostos foram realmente alcançados e que 0 software atinja o problema de ensino aprendizagem que motivou seu desenvolvimento. Uma avaliação criteriosa de um software educacional é fundamental antes de ser disponibilizado à população alvo $^{(6)}$

A avaliação pode ser definida como um processo contínuo usado para determinar se os objetivos do programa foram alcançados, identificar as razões para o desempenho observado do usuário, e identificar as partes do programa que precisam ser modificadas ${ }^{(1)}$.

Para realizar a avaliação do programa, fizemos uma adaptação do Modelo de três estágios para o desenvolvimento de
Programas $\mathrm{CAl}^{(1)}$. Assim, a fase de avaliação foi dividida em três etapas: etapa 1, 2 e 3.

A etapa 1 teve por finalidade avaliar a execução do software verificando incompatibilidades. Os avaliadores dessa etapa foram analistas de sistemas e membros da equipe de desenvolvimento do programa. Na etapa 2 foram avaliadas a qualidade do conteúdo, dos objetivos e as estratégias utilizadas, sendo realizada por especialistas no conteúdo em questão. A terceira e última etapa de avaliação foi realizada por alunos de graduação em enfermagem, mães e professoras de escola infantil, sendo avaliada a eficácia do mesmo quanto à adequação instrucional, estética e adequação do software.

Todos os participantes das etapas de avaliação assinaram um "termo de consentimento de participação em pesquisa livre e esclarecido". Para cada etapa de avaliação foi utilizado um instrumento específico, segundo as adaptações do modelo, com vistas a atingir os objetivos propostos para cada uma delas.

\section{RESULTADOS}

A avaliação é um processo contínuo e que nunca estará completa. Após o término de um software educacional e sua disponibilização para uso, o feedback dos usuários pode fornecer idéias para melhorias que poderiam ser incorporadas em versões subseqüentes do programa.

De forma geral, o software "A criança e o medicamento" foi bem aceito pelos avaliadores, emergindo comentários positivos sobre o trabalho desenvolvido. Em cada etapa da avaliação vários aspectos eram analisados por meio de itens classificados pelos avaliadores de acordo com a seguinte escala: insatisfatório, razoável, satisfatório, excelente.

Etapa 1

Participaram desta avaliação três analistas de sistemas, 0 programador do software e o técnico audiovisual, sendo que um dos analistas realizou três testes, resultando portanto, em sete avaliações. Nessa etapa foram avaliados três aspectos: tempo de resposta, qualidade das telas e qualidade da interface do software em vários tipos de equipamentos (variando de um processador 486 133Mhz e sistema operacional Windows 95 à um processador PENTIUM Pro III 450Mhz e sistema operacional Windows 98). A seguir foram apresentados os itens avaliados em cada um dos aspectos acima relacionados:

a) Tempo de resposta (7 itens): iniciação do programa; utilização de memória; troca de telas; links; feedback dos testes; botões de navegação e saída do programa. 
b) Qualidade das telas (6 itens): qualidade dos vídeos; qualidade das fotos; qualidade das figuras; qualidade do som; qualidade das animações e textos.

c) Qualidade da interface (11 itens): uso do espaço; formato das telas; facilidade de uso; aspecto visual; botões de navegação; uso de cores; uso de figuras; uso de vídeos; uso de animações; uso de fotos e uso de sons.

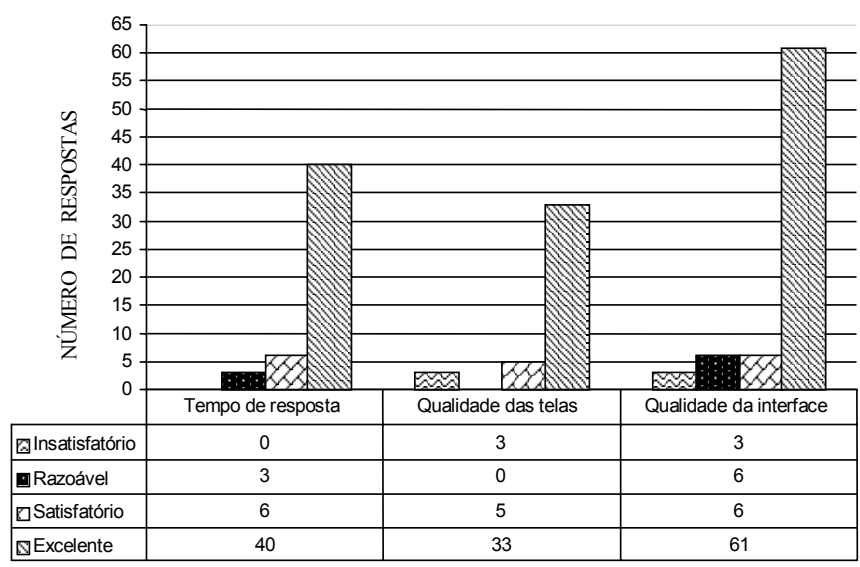

Figura 1 - Distribuição das respostas obtidas na avaliação dos aspectos: tempo de resposta, qualidade das telas e qualidade da interface do programa de acordo com os itens apresentados

Como foram realizadas sete avaliações nesta etapa, para cada aspecto obtivemos um número específico de respostas, de acordo com o número de itens avaliados, ou seja, para tempo de resposta, tivemos 49 respostas, para qualidade das telas 41 respostas, pois um avaliador não respondeu um dos itens e para 0 aspecto qualidade da interface 76 respostas, pois um avaliador não respondeu um dos itens.

Na Figura 1 apresentamos a distribuição das respostas obtidas nesta etapa da avaliação, mostrando que o tempo de resposta do software foi considerado excelente pelos avaliadores, de acordo com a maioria das respostas (40 respostas/81,64\%) obtidas para este aspecto. A maioria dos itens (33 respostas/80,49\%) analisados quanto à qualidade das telas foi considerada excelente nos testes realizados. No entanto, em três testes ( 3 respostas $/ 7,32 \%$ ), 0 item qualidade dos vídeos foi considerado insatisfatório. Isso porque estes testes foram realizados no ambiente Windows 95, que não possuía o descompactador "vids.IV5.0". Assim, acrescentamos ao software o driver referido para poder ser instalado nesses casos, com as devidas instruções.

A análise dos dados sobre a qualidade da interface do software, mostrou que a maioria dos itens (61 respostas/80,27\%) foi considerada excelente nos testes realizados. Observa-se na Figura 1 que a maioria dos itens (134 respostas/80,72\%) avaliados nos testes realizados com o software em diferentes equipamentos, teve consideração excelente, exigindo poucas modificações no produto.
Etapa 2

Esta etapa da avaliação foi realizada por três docentes e quatro enfermeiras da área de pediatria, que foram consideradas, neste estudo, como especialistas no tema do software. O instrumento utilizado abordou quatro aspectos: apresentação e qualidade do conteúdo, qualidade audiovisual, qualidade dos testes e adequação à população alvo. Para cada aspecto foram avaliados os seguintes itens:

a) Apresentação e qualidade do conteúdo (7 itens): abrangência; atualização; vocabulário; seqüência instrucional dos tópicos; apresentação dos conceitos; objetivos dos tópicos - relação entre as tarefas instrucionais e o conteúdo dos tópicos.

b) Qualidade audiovisual (5 itens): figuras; vídeos; animações; fotos e locução.

c) Qualidade dos testes (4 itens): apresentação; enunciado; feedback às respostas e associação ao conteúdo do software.

d) Adequação à população alvo (5 itens): aluno de curso de enfermagem; docente de enfermagem; mães/pais; professores de escola infantil e profissionais de enfermagem.

Nesta etapa foram realizadas sete avaliações, portanto, obtivemos 49 respostas para 0 aspecto apresentação e qualidade do conteúdo, 35 para qualidade audiovisual, 28 para qualidade dos testes e 35 para adequação à população alvo, perfazendo um total de 147 respostas. A análise dos dados mostrou que a maioria dos itens (109 respostas/74,15\%) recebeu consideração excelente (Figura 2) e nenhum dos itens foi considerado insatisfatório ou razoável.

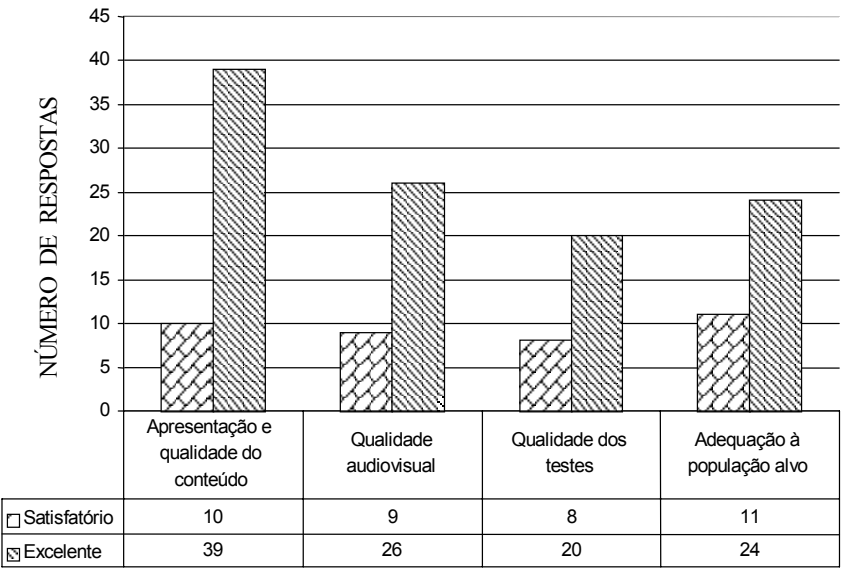

Figura 2 - Distribuição das respostas obtidas na avaliação dos aspectos: apresentação e qualidade do conteúdo; qualidade audiovisual; qualidade dos testes e adequação à população alvo de acordo com os itens apresentados

$\mathrm{Na}$ Figura 2 observa-se que a maioria dos itens (39 respostas/79,59\%) avaliados foi considerada excelente pelas especialistas quanto à apresentação e qualidade do conteúdo. 
Quanto à qualidade audiovisual e qualidade dos testes, a análise dos dados mostrou que a maioria dos itens (26 respostas $/ 74,29 \%$ e 20 respostas $/ 71,43 \%$, respectivamente) foi considerada excelente pelas avaliadoras.

No que tange ao aspecto adequação à população alvo, a maioria dos itens (24 respostas/68,57\%) foi classificada como excelente e $31,43 \%$ das respostas (11) consideraram este aspecto como satisfatório. Além dos dados apresentados na Figura 2, a avaliação positiva do software pelas especialistas pode ser observada também pelos seguintes comentários: Excelente trabalho tanto no conteúdo quanto na qualidade audiovisual e população alvo. Acredito que possa ser de grande utilidade prática no ambiente de trabalho com equipe de enfermagem e mães acompanhantes. A apresentação do software atingiu seu objetivo pois foi apresentado de forma clara, com uma linguagem acessivel, com conteúdo de extrema necessidade para a população destinada. Destaco ainda, a importância das fotos e vídeos apresentados para o completo entendimento dos tópicos em questão.

Esses relatos apontaram a aceitação do software por parte dessas especialistas não apenas como um recurso a mais para 0 ensino de enfermagem, mas como um recurso inovador e motivador, que poderá ser útil tanto em nível médio, como superior e educação continuada.

Etapa 3

Participaram sete alunos do último semestre do curso de Enfermagem da Universidade Federal de São Carlos, cinco mães e cinco professores de escolas infantis.

Essa etapa teve por objetivo de avaliar a eficácia do software quanto à adequação instrucional, adequação estética e adequação do programa. A seguir são apresentados os itens avaliados em cada aspecto:

a) Adequação instrucional (9 itens): apresentação do menu de entrada; conteúdo; tamanho do conteúdo nos tópicos; clareza no texto; seqüência instrucional dos tópicos; apresentação dos procedimentos técnicos nos tópicos; oportunidade de instrução individual; interatividade - relação usuário-máquina e motivação.

b) Adequação estética ( 7 itens): uso do espaço da tela; qualidade dos sons; qualidade das figuras; qualidade dos vídeos; qualidade das animações; apresentação visual dos testes e feedback (qualidade do reforço às respostas).

c) Adequação do programa (5 itens): facilidade na execução do programa; entrada no programa; apresentação das instruções de uso; testes de avaliação da aprendizagem e saída do programa.

Como foram realizadas dezessete avaliações nesta etapa, para o aspecto adequação instrucional tivemos 153 respostas, para adequação estética 119 respostas e para adequação do programa 85 respostas, como pode ser observado na Figura 3 .

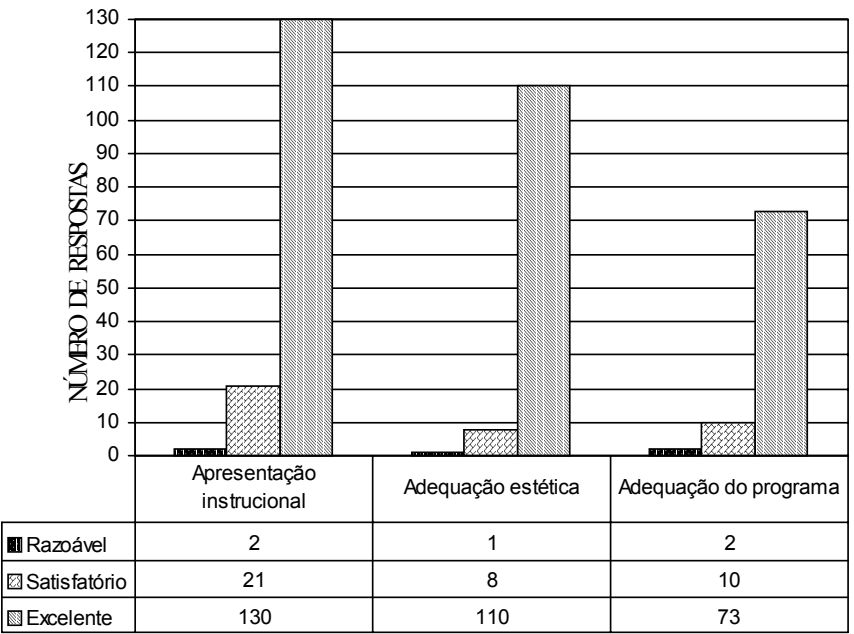

Figura 3 - Distribuição das respostas obtidas na avaliação dos aspectos: adequação instrucional; adequação estética e adequação do programa de acordo com os itens apresentados

A análise dos dados apresentados na Figura 3 relativos ao aspecto adequação instrucional do software, mostrou que dois itens foram considerados razoáveis: tamanho do conteúdo nos tópicos e interatividade - relação usuário-máquina. Entretanto, outros avaliadores fizeram comentários positivos com relação a esses itens. O trabalho ficou muito bem e interessante, principalmente por solicitar a participação do usuário, isso auxilia na motivação do mesmo.

A adequação estética do software foi considerada excelente pelos avaliadores (110 respostas/92,44\%) como pode ser observado na Figura 3. Entretanto, uma das alunas considerou a qualidade dos sons apenas razoável, relatando que estava com muita falha, sendo o único comentário desse tipo. Quanto à adequação do programa, a maioria dos itens (73 respostas $/ 85,88 \%$ ) foi considerada excelente. 0 item "entrada no programa" foi avaliado como razoável por dois alunos, talvez porque essa avaliação esteja relacionada com o tipo de equipamento onde o software foi preparado. Essa avaliação mostra que o objetivo foi alcançado no sentido de poder oferecer um produto novo, diferente daqueles existentes para abordar 0 assunto em questão. Os comentários realizados pelos avaliadores nos incentivam a continuar o processo de aperfeiçoamento do programa, que sabemos ser indispensável para o desenvolvimento de novas estratégias de ensino: Material muito bem elaborado, visual, teoria, esforço reconhecido, parabéns. Gostei muito, está muito agradável e instrutivo. Contém informações importantes para as mães e pais.

As avaliações realizadas foram de grande valia e proporcionaram alertas para modificação de alguns aspectos do software, importantes para sua futura disponibilização. Nossa meta visa a melhoria contínua deste processo de avaliação, acompanhando e incorporando contribuições significativas. Sabemos que apesar de existir uma produção cada vez maior de materiais desse tipo, o seu 
emprego na educação não tem sido avaliado de forma sistemática e enfrenta alguns desafios ${ }^{(7)}$.

Várias pesquisas precisam ser desenvolvidas sobre avaliação de software educacional, área sabidamente recente e aberta ainda a inúmeros estudos ${ }^{(6)}$. Nossa preocupação no momento em que terminamos o software, entretanto, foi realizar uma avaliação junto a peritos e população alvo baseando-nos na literatura disponível. Como resultado, pudemos verificar a aceitação do software por essa população e acreditamos que muito ainda poderá ser realizado em termos de avaliação, para o aprimoramento do programa a fim de que possamos contribuir com o oferecimento desse recurso para 0 ensino de enfermagem.

\section{CONSIDERAÇÕES FINAIS}

A utilização de recursos computacionais como ferramenta efetiva no processo ensino-aprendizagem e a implementação deste instrumento no ambiente educacional vem se confirmando desde a pré-escola até a universidade. Muitos docentes e alunos apresentamse ainda resistentes quanto ao emprego desses recursos, pois para

\section{REFERÊNCIAS BIBLIOGRÁFICAS}

1. Price RV. Computer-aid instruction: a guide for authors. Pacific Grove: Brooks/Cole Publishing Company; 1991.

2. Cassiani SHB. Um salto para o futuro no ensino da administração de medicamentos: desenvolvimento de um programa instrucional auxiliado pelo computador. [dissertação]. Ribeirão Preto (SP): Escola de Enfermagem de Ribeirão Preto/USP; 1998

3. Rocha MT. Construção de uma base de dados particular informatizada. Rev Paul Enfermagem 1992; 11(2):64-8.

4. Zem-Mascarenhas SH, Carvalho EC. Toque terapêutico: análise da produção do conhecimento utilizando uma base de dados informatizada. Rev Min Enfermagem 1998; 2(2):98-103. poder utilizá-los são necessários alguns conhecimentos básicos sobre informática, o que pode tornar-se um transtorno para os avessos à tecnologia computacional, sempre em constante transformação.

Não podemos nos iludir em acreditar que a informática é a solução para os problemas educacionais ou sociais, ou que é o único caminho a ser trilhado. Temos que encarar a informática como mais uma ferramenta que pode ser de utilidade para a educação. A utilização de recursos hipermídia em ambientes de ensino oferece uma maior flexibilidade de apresentação das informações aos usuários. $\mathrm{E}$, além disso, também os estimula a aprender, devido a recursos audiovisuais normalmente disponíveis ${ }^{(8)}$.

Após analisarmos as avaliações realizadas com o programa pudemos comprovar nossa contribuição quanto à proposta inicial de desenvolvimento de um programa de ensino auxiliar para a enfermagem, e a partir de agora a enfermagem pediátrica tem a sua disposição um novo recurso para o ensino de administração de medicamentos a crianças. Ainda não podemos prever o que o futuro nos reserva, mas a tecnologia computacional promete para o presente muitos desafios excitantes e oportunidades inimagináveis para 0 futuro.
5. Gagné RM. Princípios essenciais da aprendizagem para o ensino. Porto Alegre(RS): Editora Globo; 1980.

6. Borges PRT. Qualidade de software educacional: critérios para validação de treinamentos multimídia utilizados em educação à distância. Tecnol Educacional 1998; 26(140):11-7.

7. Silva CMT da. Hipermídia na educação: potencialidades e desafios. Tecnol Educacional 1998; 26(140):18-23.

8. Freire MEP. O sistema tutor de um ambiente inteligente para treinamento e ensino. [dissertação]. São Carlos (SP): Instituto de Ciências Matemáticas de São Carlos/USP; 1998. 